\title{
A Simple Technique for Removal of Interlocking Screw from an Intramedullary Nail: A Case Report
}

\author{
Sumanth Madhusudan Prabhakar ${ }^{1}$, Joshua Decruz ${ }^{1}$, Wee Liang Hao James ${ }^{1}$, \\ Remesh Kunnasegaran ${ }^{1}$
}

Learning Point of the Article:

Where conventional methods of screw removal are not successful, it is prudent to consider other techniques early so as to minimise surgical complications and surgical time.

\section{Abstract}

Introduction: Difficulties encountered during removal of implants present a common technical challenge in orthopedic surgery, for which a number of factors have been implicated. A variety of techniques and instruments have been used to overcome this. However, some of these may prove to be time consuming, expensive, and inaccessible to many surgical setups. We describe a technique used for the removal of a jammed interlocking screw from an intramedullary nail that allows for minimal damage to the hardware, bone, and surrounding soft tissue, with the added advantage of being relatively quick and technically uncomplicated with the use of simple instruments.

Case Report: We describe the case of an 81-year-old female with a history of surgical fixation for a left femur intertrochanteric fracture, who presented with groin pain 13 months post-fixation. Radiographs were suggestive of avascular necrosis of the femoral head with resultant cut-in of the blade, and the patient was eventually taken up for the removal of implants and total hip replacement. Intraoperatively, difficulties were encountered in the removal of the distal interlocking screw, with failure of conventional techniques initially. A high-speed burr was then employed to shape the screw head so as to achieve better grip with extraction devices, which facilitated smooth removal.

Conclusion: We describe a simple method for difficult screw removal involving the use of a high-speed burr and vise grip pliers. This technique provides a quick and inexpensive option with commonly available surgical tools and may be considered when encountering difficulties with screw extraction.

Keywords: Trauma, revision surgery, interlocking screw, screw removal.

\section{Introduction}

Implant removal may be clinically indicated in several situations, including implant-related pain, infection, hardware failure, revision for non-union or malunion, and patient's request. Difficulties in hardware removal, in particular the removal of jammed or damaged screws, present a common surgical challenge. A variety of factors have been implicated in this, including the presence of cold welding and/or crossthreading between threads in the screw head and screw hole, and high levels of osteointegration between the implant and bone $[1,2,3]$.
A variety of techniques have been described to aid in the removal of such screws. The use of conical extraction screws, hollow reamers, extraction bolts, modular devices, carbide drill bits and screw removal kits has all been detailed in literature [4, 5].

Although some of these techniques may prove to be successful, they can prove to be time consuming, costly, and inaccessible to many surgical setups.

We describe a novel technique used for the removal of a jammed interlocking screw from an intramedullary nail that allows for minimal damage to the hardware, bone, and surrounding soft
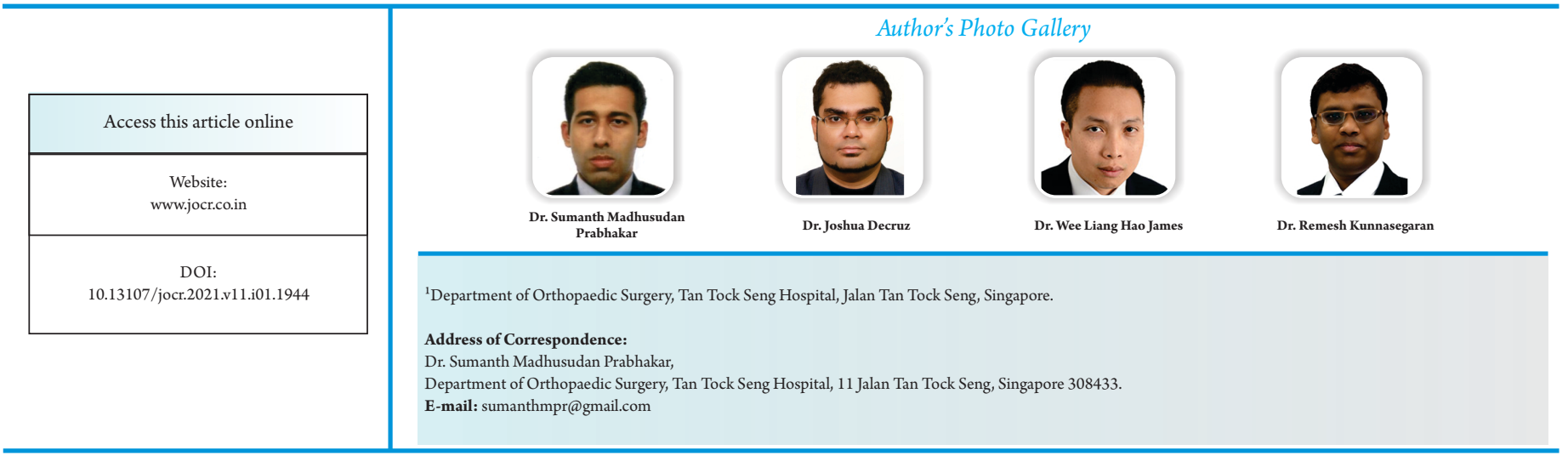

Journal of Orthopaedic Case Reports | pISSN 2250-0685 | eISSN 2321-3817 | Available on www.jocr.co.in | doi:10.13107/jocr.2021.v11.i01.1944 This is an Open Access article distributed under the terms of the Creative Commons Attribution Non-Commercial License (http://creativecommons.org/licenses/by-nc/3.0) which permits unrestricted non-commercial use, distribution, and reproduction in any medium, provided the original work is properly cited. 


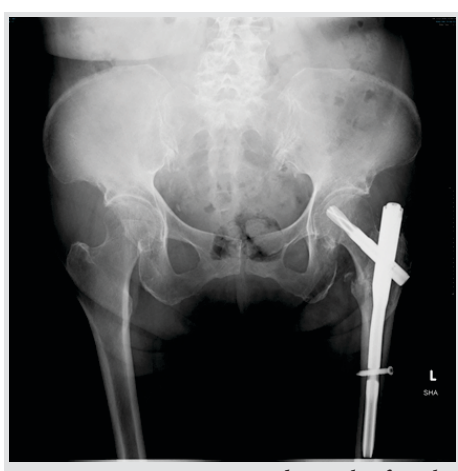

Figure 1: Post-operative radiograph after the index operation with PFNA device, 3 years prior to current presentation

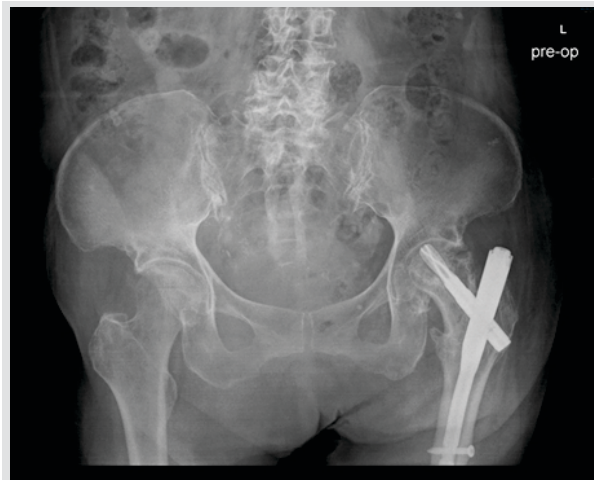

Figure 2: Pre-operative radiograph displaying cut-in of the PFNA blade with AVN of the femoral head.

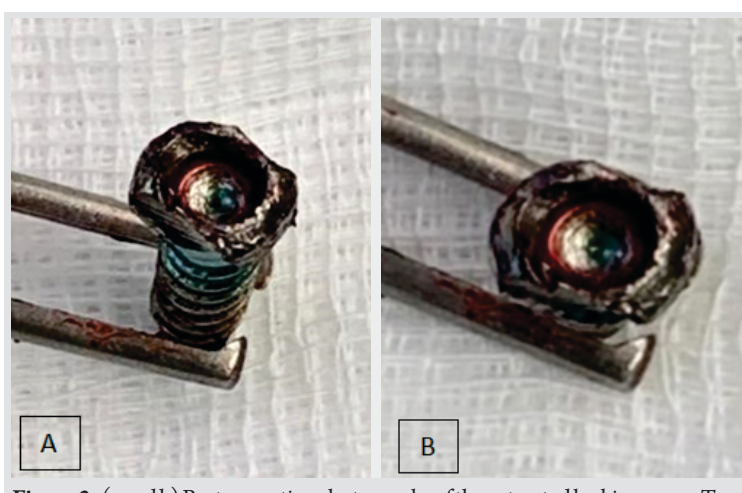

Figure 3: (a and b) Post-operative photographs of the extracted locking screw. Two sides of the screw head were bured burred off to create a more rectangular shape. The rest of the screw was observed to be structurally intact. tissue, with the added advantage of being relatively quick and technically uncomplicated with the use of simple instruments.

\section{Case Report}

We present a case of an 81-year-old female who had surgical fixation performed for a comminuted four part left intertrochanteric fracture 3 years previously at the same institution with an intramedullary device. After an initial uneventful post-operative recovery (Fig. 1), she returned 13 months post-fixation with groin pain, with radiographs confirming avascular necrosis of the femoral head with resultant cut-in of the blade (Fig. 2). Having failed initial conservative management and with worsening function and pain, the patient eventually opted for surgery.

Pre-operative computed tomography scan of the left hip was performed, revealing a healed intertrochanteric fracture without any peri-implant fractures, as well as flattening and deformity of the femoral head with degenerative changes in the hip joint suggestive of avascular necrosis. The patient was consented for removal of implants and total hip replacement and underwent the surgery 38 months post-index procedure.

Intraoperatively, difficulty was encountered during the removal of the distal interlocking screw. This was likely secondary to a divergent trajectory of the bolt resulting in cold welding to the screw to the intramedullary nail. Attempts were made at removal using vise grip pliers, which failed due to insufficient purchase on the screw head. Subsequently, the Synthes broken screw set was utilized to attempt screw removal, with conical extraction screws as well as extraction bolts. This was also unsuccessful. With every attempt at screw removal, the screw head became progressively more deformed, making subsequent removal increasingly difficult.

We then decided to use a different approach for the screw removal. A high-speed burr was used to burr around the screw, helping to "square" the circular screw head so as to achieve a better grip on the screw head with the extraction devices. With this, it was now possible to achieve adequate grip on the screw head with vise grip pliers, and the screw was then removed in entirety with relative ease. The screw was observed to be otherwise intact with no broken sections (Fig. 3a, 3b).

The remainder of the procedure was uneventful and a successful total hip replacement was performed (Fig. 4).

\section{Discussion}

A number of contributing factors have been identified in the difficult removal of screws, including jammed and broken screws $[1,2,3,4,5]$. Common reasons include (1) jamming of the screw head in the screw hole due to cold welding or crossthreading, (2) bony growth and osteointegration, and (3) stripping of the recess of the screw heads while using a screwdriver. Other factors discussed include incorrect angle of insertion during the index operation, excessive tightening of the screw head, and lack of use of torque limiting screwdrivers. Among these factors, it is possible that incorrect angle of insertion during the index operation contributed to the difficulty encountered in screw removal in this case (Fig. 5).

Bony overgrowth in particular is a significant occurrence with the presence of titanium implants, due to their high

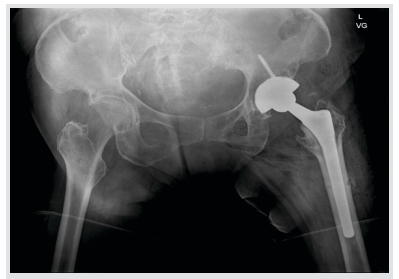

Figure 4: Post-operative radiograph after removal of implants and total hip arthroplasty.

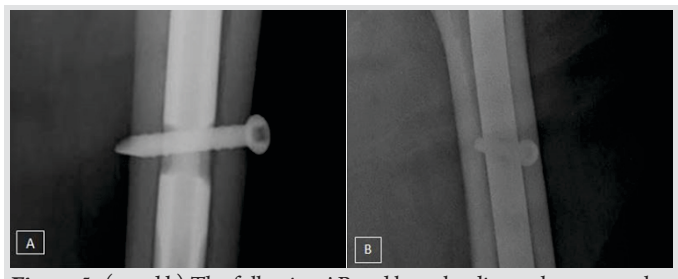

Figure 5: $(\mathrm{a}$ and $\mathrm{b})$ The following $\mathrm{AP}$ and lateral radiographs suggest that insertion of the distal interlocking screw at an oblique angle may have been a factor in difficult removal.
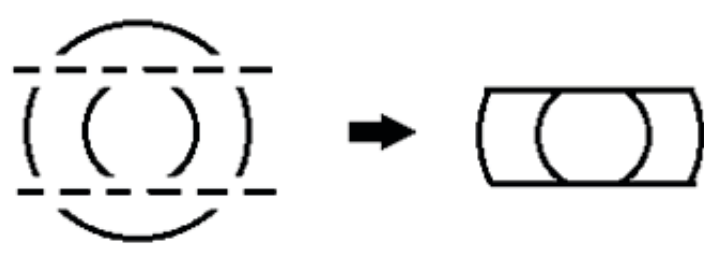

Figure 6: Increasing the contact area of the screw head with the extraction too allowed for a higher grip force to be applied to the screw. 
biocompatibility, leading to increased levels of osteointegration [6].

The potential complications arising from screw removal include breakage of screw, implant retention, increased soft tissue and bony damage, and increased operating time [7]. It is important that these factors are considered in the selection of a technique for screw removal, as such complications can easily add to surgical time and cost, and increases the overall morbidity risk of the procedure. In our case, removal of the screw was a prerequisite prior to the insertion of a prosthetic implant; hence, safe and efficient screw removal was a necessity.

The technique we applied provided a clear mechanical advantage during the screw removal process. By burring two ends of the screw head, we increased contact area of the screw head by burring it to a rectangular profile, thereby enabling a higher grip force on the screw (Fig. 6).

Several techniques for screw removal are described in the existing literature. A number of these techniques are targeted toward the removal of jammed locking screws in locking plates. Several authors describe techniques involving conical extraction screws, extraction bolts, hollow reamers, and carbide drillbits.

Kumar and Dunlop [5] described a technique that involves using a high-speed disc to make radial cuts from the locking plate edge to the screw hole, with an osteotome wedged in the cut. This increased the circumference of the threaded portion of the screw hole and released the screw head, facilitating removal of screw with the conical extraction screw. This technique, however, poses a risk of damage to the screw head by the disc, thermal bone necrosis, and iatrogenic bony injury. In addition, such sophisticated devices may not be readily accessible in many operating theatres.

Park and Jang [8] described a technique utilizing a screw extractor (a non-medical instrument) to remove locking screws with a damaged screw head. While inexpensive and quick to perform, there is likely to be a large variation in the quality and reliability of non-medical instruments, which may be a barrier to more widespread usage of non-medical instruments in general. Kumar et al. [9] detailed a technique involving the use of a novel T-shaped screw extraction device to remove jammed locking screws.

The technique utilized in this study utilizes standard instruments which should be readily available in most surgical setups. It may be performed rapidly while also avoiding extensive bone loss and soft-tissue damage. Furthermore, the technique is universally applicable and is not limited by screw size, shape, or design.

A previous report by Iwata et al. [10] describes a "parallel plate technique" similar to ours, used in the presence of a damaged screw head. This technique involved applying a high-speed burr to opposite sides of the screw head, forming parallel faces located away from the recess of the screw head. This allowed the two faces to be grasped with locking pliers and the implant to be unscrewed with gentle upward traction. The similar concepts employed suggest that such methods may be indeed have a high degree of applicability.

\section{Conclusion}

We describe a simple method for difficult screw removal involving the use of a high-speed burr and vise grip pliers. This technique provides a quick and inexpensive option with commonly available surgical tools and may be considered when encountering difficulties with screw extraction.

\section{Clinical Message}

Difficulties may be encountered in the removal of screws in the context of revision surgery. Where conventional methods of screw removal are not successful, it is prudent to consider other techniques early so as to minimize surgical complications and surgical time.

\section{References}

1. Georgiadis GM, Gove NK, Smith AD, Rodway IP. Removal of the less invasive stabilization system. J Orthop Trauma 2004; 18:562-4.

2. Ehlinger M, Adam P, Simon P, Bonnomet F. Technical difficulties in hardware removal in titanium compression plates with locking screws. Orthop Traumatol Surg Res 2009;95:373-6.

3. Hamilton P, Doig S, Williamson O. Technical difficulty of metal removal after LISS plating. Injury 2004;35:626-8.
4. Hak DJ, McElvany M. Removal of broken hardware. J Am Acad Orthop Surg 2008;16:113-20.

5. Kumar G, Dunlop C. Case report: A technique to remove a jammed locking screw from a locking plate. Clin Orthop Relat Res 2011;469:613-6.

6. Moroni A, Faldini C, Rocca M, Stea S, Giannini S. Improvement of the bone-screw interface strength with hydroxyapatite-coated and titanium-coated AO/ASIF cortical screws.J Orthop Trauma 2002;16:257-63. 
7. Sanderson PL, Ryan W, Turner PG. Complications of metalwork removal. Injury 1992;23:29-30.

8. Park WR, Jang JH. An inexpensive and rapid method for removal of multiple stripped locking screws following locking plating: A case report. Int J Surg Case Rep 2019;57:134-7.
9. Kumar A, Saini M, Jameel J, Khan R. "Turn the tail, not the head": A simple, quick and inexpensive technique for the safe removal of jammed/stripped locking screws. Eur J Orthop Surg Traumatol 2020;30:1119-23.

10. Iwata T, Nozawa $S$, Maeda M, Akiyama $H$. New technique for removal of screws with damaged heads. Orthopedics 2017;40:e911-4.

\section{How to Cite this Article}

Conflict of Interest: Nil

Source of Support: Nil

Consent: The authors confirm that informed consent was obtained from the patient for publication of this case report
Prabhakar SM, Decruz J, James WLH, Kunnasegaran R. A Simple Technique for Removal of Interlocking Screw from an Intramedullary Nail: A Case Report. Journal of Orthopaedic Case Reports 2021 January; 11(1):12-15. 